\title{
Contribuciones al Desarrollo Forestal de Costa Rica
}

\section{Contributions to Forest Development of Costa Rica}

\section{Elizabeth Arnáez-Serrano¹, Ileana Moreira-González²}

Arnaéz-Serrano, E; Moreira-González, I. Contribuciones al Desarrollo Forestal de Costa Rica. Tecnología en Marcha.

Especial 2019. 25 Aniversario del Centro de Investigación en Biotecnología. Pág 36-40.

doi) https://doi.org/10.18845/tm.v32i9.4625

1 Bióloga. Escuela de Biología. Instituto Tecnológico de Costa Rica. Costa Rica. Correo electrónico: earnaez@tec.ac.cr 


\title{
Palabras clave
}

Especies forestales; semillas forestales; anatomía vegetal,; autoecología.

\begin{abstract}
Resumen
El presente artículo muestra una revisión histórica de un grupo de funcionarios de la Escuela de Biología del Instituto Tecnológico de Costa Rica (TEC), en conjunto con investigadores de la Escuela de Ingeniería Forestal del TEC y de las universidades estatales de Costa Rica, que incluyeron estudios relacionados con el manejo de semillas forestales, manejo en vivero rescate de especies, anatomía vegetal, autoecología, y establecimiento en campo. Estos proyectos permitieron establecer una línea de trabajo creciente en otros campos del estudio de las especies con uso forestal.
\end{abstract}

\section{Keywords}

Forest species; forest seeds; plant anatomy; autoecology.

\begin{abstract}
This article shows a historical review of a group of officials from the School of Biology of the Technological Institute of Costa Rica (TEC), in conjunction with researchers from the Forest Engineering School of TEC and other state universities of Costa Rica, which included studies related to the management of forest seeds, nursery management, rescue of species, plant anatomy, autoecology, and establishment in the field. These projects allowed to establish a growing line of work in other fields of the study of species with forest use.
\end{abstract}

\section{Introducción}

En el año 1985, el área de Biología adscrita a la Escuela de Química, se incorporó a una serie de estudios en el tema de especies forestales con las investigaciones de Silvana Alvarenga en el tema de Swietenia macrophylla (Caoba), Benjamín Mora en Enterolobium cyclocarpum (Guanacaste) y posteriormente en los que ya se realizaban en la Escuela de Ingeniería Forestal con un aporte biológico al tema de la investigación en semillas de especies forestales, gracias a la experiencia generada por las Biólogas Elizabeth Arnáez en el estudio de Cedrela odorata (cedros amargo) [1] e lleana Moreira en los estudios de germinación de Vochysia guatemalensis (palo de mayo).

Esto permitió establecer una línea de trabajo sólida en estos temas considerando que la semilla es el medio natural de dispersión, propagación y perpetuación de la mayoría de las especies y constituye hasta el momento, la estructura menos conocida de las plantas superiores y que estudios en este tema darían pie a la obtención de financiamiento con mayor facilidad.

En un primer proyecto en conjunto con los ingenieros Freddy Rojas y Gustavo Torres de la Escuela de Ingeniería Forestal, se utilizaron semillas de diez especies forestales nativas de altura (1200-2900 m.s.n.m.), a las cuales se les describió la morfología en donde se consideraron algunos aspectos como germinación, desarrollo de plántula, fenología y manejo en general, las especies estudiadas fueron: Brunellia costarricenses (cedrillo), Prumnopitys standleyi (ciprecillo), Ulmus mexicana (tirrá), Cornus disciflora (lloró), Cedrella tonduzii (cedro), Alfaroa costarricenses (gaulín), Quercus costarricenses (roble), Prunus annularis (duraznillo), Magnolia poasana (Magnolia) y Alnus acuminata (jaul) [3], [4], [5]. 
También se trabajó con especies de las zonas bajas de Costa Rica, las cuales fueron: Calophyllum brasiliensis (Guttiferae), Cordia alliodora (Boraginaceae), Goethalsia meiantha (Tiliaceae), Hieronyma oblonga (Euphorbiaceae), Stryphnodendron microstachyum (Leguminosae), Terminalia amazonia (Combretaceae), Vochysia ferruginea (Vochysiaceae), Vochysia guatemalensis (Vochysiaceae) y Zanthoxylum mayanum (Rutaceae) [6]. Se realizaron disecciones para analizar las características morfológico-anatómicas internas y externas de las diferentes especies. La descripción se hizo del material recién colectado, así como del procesado en microscopía de luz (con parafina) y microscopía electrónica de barrido en [7].

Para entonces se vinculó la Escuela de Biología con el proyecto a nivel país COSEFORMAGTZ donde participaron además de Moreira y Arnáez, otros investigadores de la Escuela de Ingeniería Forestal tales como Olman Murillo, Marvin Castillo, Lucía Rodríguez y Dagoberto Arias. Los resultados de las investigaciones de este período 1990-1997 quedaron plasmadas en material didáctico elaborado para talleres con productores en el tema de plantaciones mixtas y domesticación de especies nativas maderables tales como: Almendro amarillo (Dipteryx panamensis) [8], Botarrama (Vochysia ferruginea), Cebo (Vochysia guatemalensis), Lagarto amarillo (Zanthoxylum mayanum), Pilón (Hyeronima oblonga) y Vainillo (Stryphnodendron excelsum). Los trabajos comprendían no solo estudios morfológicos de semillas, sino además se incorporó la metodología para determinar el comportamiento fenológico de las especies. Lo que permitió comenzar a medir el impacto del cambio climático en el comportamiento de las especies forestales nativas.

Se incorporaron después los estudios relacionados con la distribución de las raíces y su vinculación con factores ambientales, como temperatura, humedad relativa, precipitación y radiación solar, tipo, textura y composición química del suelo, entre otros. Todos estos parámetros básicos para poder hacer uso de especies nativas en modelos de plantación. Específicamente para especies como el pilón (Hieronyma alchornoides) [9], [10], botarrama (Vochysia ferruginea) [11] y cebo (Vochysia guatemalensis) [12]. Otros estudios más específicos fueron realizados en la Biología Reproductiva de especies forestales nativas de la Zona Huetar Norte de Costa Rica en Ficus insipida (Chilamate); Sclerolobium costarricense (tostao) y Brosimum costaricanum (ojoche).

Los resultados obtenidos mostraron que la metodología desarrollada tenía un gran potencial para el rescate de la variación genética y reproducción de especies forestales en peligro de extinción, haciendo posible su reintroducción en ecosistemas donde hayan sufrido una fuerte erosión genética o se hayan extinguido. También sirve de base para programas de domesticación y conservación mediante su cultivo en diversos agroecosistemas y programas de mejora genética.

El estudiar la biología estructural de las semillas es importante para comprender aspectos como por ejemplo los síndromes de dispersión, la depredación de las mismas, la manipulación de las semillas para sus diferentes usos, entre otros. El describir las semillas aporta un criterio más amplio para elegir un tratamiento pregerminativo adecuado, así como un manejo óptimo en vivero de especies de las cuales se conocía muy poco y que ahora se pueden manipular para la mejora del material vegetal que se usa para reforestación [6].

EI CIB desde sus inicios aportó a la problemática del desarrollo forestal de Costa Rica desde dos puntos de vista, uno es la tasa de deforestación anual que tiene el país (entre el 2005 y el 2015 Guanacaste perdió 63.050 hectáreas de bosque) [13] y por otro los planes de reforestación que a su vez involucran la elección de especies, zonas óptimas de plantación, manejo de las especies en plantación y obtención del material para cumplir estos fines [3], [4], [6]. Su papel permitió, por lo tanto, contribuir a mejorar los procesos de selección de especies forestales y apoyar en los planes de reforestación en Costa Rica. 
Otra novedad en la que el CIB participó fue con la interpretación de los estudios empleando diferentes instrumentos tales como: microscopía electrónica de barrido y microscopía de luz con inmersión del material en parafina, así como estudiar el proceso de germinación y desarrollo de plántula, lo que brindó nuevos aportes en aspectos de comportamiento de las diferentes especies [3], [4], [5], [6], [7], [14]. En Costa Rica, son pocos los estudios realizados en este campo; sin embargo, son las instituciones de educación superior las que más han colaborado en este aspecto, entre ellas: Universidad de Costa Rica, Instituto Tecnológico de Costa Rica, Universidad Nacional, Centro Agronómico Tropical de Investigación y Enseñanza (CATIE) y Organización para Estudios Tropicales.

Después de mucho esfuerzo, con base en las investigación demostradas por la Escuela de Biología (CIB) y la Escuela de Ingeniería Forestal (CIIBI), Ilevaron al sector forestal a organizarse, debido a la necesidad inminente de una solución a mediano y largo plazo que permitiera a las diferentes zonas, recuperarse del deterioro ambiental que habían sufrido, al deterioro de la calidad de las fuentes de agua para las zonas urbanas, la erosión del suelo, pérdida de bancos de germoplasma, así como de sus bellezas escénicas naturales visitadas por otros sectores como el turismo, la academia universitaria, etc. La domesticación de las especies forestales nativas se vio como una opción para impulsar el desarrollo forestal del país.

El CIB siguió participando en proyectos con especies forestales donde se incluían ya técnicas novedosas como la crioconservación en especies ya estudiadas en los que a comportamiento se refiere, en donde la PhD. Ana Abdelnour fue la investigadora que le ha permitido figurar como un Centro de Investigación con un gran valor en este tema.

Entre las alianzas de Centros de Investigación, las investigadoras Arnáez y Moreira unieron esfuerzos en un proyecto interuniversitario (UNA, ITCR, UNED, CONARE, 2007-2009), propuesto y coordinado por el INISEFOR, que tuvo como objetivo contribuir a la supervivencia y conservar la diversidad genética de seis especies en peligro crítico (Cedrela salvadorensis, Platymiscium yucatanum, Paramachaerium gruberi, Cedrela fissilis, Ruagea insignis y Gamanthera herrerae [14], [15], mediante la aplicación métodos de conservación y reproducción ex situ. Como actividad adicional del proyecto, se estableció un rodal de conservación genética de Swietenia macrophylla (caoba) mediante la donación por parte del CATIE de una colección de semillas de varias poblaciones de América Latina, trabajo liderado por el Ingeniero Eugenio Corea.

Actualmente el CIB participa en los estudios forestales que se están realizando en el Área de Conservación OSA (Costa Rica) educando en temas de su competencia a las asociaciones que funcionan en este lugar en pro de lograr un manejo forestal consciente y ateniente a las demandas actuales del mercado protegiendo la biodiversidad, coordinado por el Ing. Marvin Castillo de la Escuela de Ingeniería Forestal.

\section{Conclusiones}

Se pudo comprobar en campo las importantes limitantes reproductivas en varias especies y poblaciones, como por ejemplo la ausencia de floración, que pocos árboles producen semilla viable, algunos en muy pequeñas cantidades o solamente en algunos años. Es notoria la escasez casi total de individuos árboles jóvenes en muchos de los individuos estudiados. Entre las posibles razones de esta situación están: cambio climático, erosión genética, aislamiento y endogamia, ausencia de polinizadores y/o de dispersores de las semillas, falta de hábitat adecuado para las plántulas, etc. 


\section{Referencias}

[1] Arnáez, E. y Flores, E. 1988. Características de la madera de Cedrela odorata L. (cedro amargo), Meliaceae., en Costa Rica. Revista Biología Tropical, 36 (1): 67-75.

[2] Moreira, I. y Arnáez, E. 1990. Estudio preliminar sobre la autoecología de Vochysia hondurensis. Rev. Tecnología en Marcha, 10 (3): 29-34.

[3] Rojas, F.; Torres, G.; Arnáez, E. y Moreira, I. 1992. Especies Forestales Tropicales. Cuadernos Científicos y Tecnológicos 1-10. Editorial Tecnológica, Costa Rica.

[4] Arnáez, E. y Moreira, I. 1992. Estudio morfológico de semillas de especies forestales de altura. Tecnología en Marcha 11(3): 67-72.

[5] Bozo, J. (editor). 2002. Tropical Tree, seed manual. United States Department of Agriculture Forest Service.

[6] Moreira, I. y Arnáez, E. 1994. Morfología de las estructuras reproductoras y germinación de nueve especies forestales nativas de Costa Rica. Revista Biología Tropical 42 (2): 73-82

[7] Moreira, I y Arnáez, E. 2000. Estudio morfológico de semillas de especies forestales nativas. Rev. Tecnología en Marcha (ITCR) 13(Especial):76-80

[8] Baltazar H. ; Moreira, I. ; Arnáez, E. ; Sánchez, E. 2001. Estudio morfológico de diferentes estadios ontogénicos de flor, fruto y semilla de Dipteryx panamensis (almendro) Tecnología en Marcha 14(1): 124-132.

[9] Arnáez, E. y Moreira I. 2004. Estudio de raíces en Hieronyma alchorneoides Allemao (pilón) en Sarapiquí, Heredia, Costa Rica. Kurú 3(9) 31 diciembre.

[10] Moreira, I. y Arnáez, E. 2008. Algunos aspectos de la morfología radicular de Hieronyma alchornoides (pilón). Revista Tecnología en Marcha 22(1): 66-76.

[11] Arnáez, E. y Ortiz, R. 2010. Estudio radicular de Vochysia ferruginea (botarrama) en plantación y condiciones naturales. Costa Rica. Revista Tecnología en Marcha. 23(1): 9-18.

[12] Moreira, I. y Arnáez, E. 2007. Estudio radicular de Vochysia guatemalensis en Sarapiquí Heredia, CR. Tecnología en Marcha 20(1): 3-12.

[13] Méndez, A. Logros en la cobertura forestal enfrentan nuevas amenazas Programa Estado de la Nación. 2018. https://www.estadonacion.or.cr

[14] Corea Arias, E.; Arnáez-Serrano, E., Moreira-González , I., Castillo-Ugalde, M. 2016.Situación de nueve especies forestales en peligro crítico de extinción en Costa Rica. Revista Forestal Mesoamericana Kurú 13(33): 36-46

[15] Moreira, I; Arnáez, E., Sánchez, E. 2015. Algunos aspectos del desarrollo ontogénico de Platymiscium pinnatum var. polystachium (Jacq.) Dugand (Cristóbal). Tecnología en Marcha 17(2): 3-12. 